\title{
The application of the "KISS principle" for the treatment of type A acute aortic dissection: is this always right?
}

\author{
Luca Di Marco, Alessandro Leone, Giacomo Murana, Davide Pacini \\ Cardiac Surgery Unit, Cardio-Thoracic-Vascular Department, S. Orsola Hospital, University of Bologna, Bologna, Italy \\ Correspondence to: Luca Di Marco, MD, PhD. Cardiac Surgery Unit, Cardio-Thoracic-Vascular Department, S. Orsola Hospital, University of \\ Bologna, Via Massarenti, 9, 40138 Bologna, Italy. Email: luca.dimarco@aosp.bo.it; ludima08@libero.it. \\ Comment on: Chiu P, Trojan J, Tsou S, et al. Limited root repair in acute type A aortic dissection is safe but results in increased risk of reoperation. J \\ Thorac Cardiovasc Surg 2018;155:1-7.e1.
}

Submitted Aug 04, 2018. Accepted for publication Aug 28, 2018.

doi: $10.21037 /$ jtd.2018.08.132

View this article at: http://dx.doi.org/10.21037/jtd.2018.08.132

Maybe not all of us know that the "KISS" design principle which literally spells "Keep it simple stupid" derives from a wordplay conceived by the U.S. aircraft engineer Kelly Johnson in the year 1960, and the concept later became very popular within the Navy in the seventies $(1,2)$. The "KISS principle" is a design rule which basically states that systems perform best when based upon simple designs rather than more complex ones; unnecessary complexity should therefore be avoided in favor of simplicity as a basic ingredient in the engineering field.

The acronym was created by Johnson (3) who was the lead engineer of the Lockheed U-2 spy plane in the 1960s; he challenged his team of design engineers to design jet aircrafts so that even an average mechanic would be able, under combat conditions, to repair it with basic tools. The word "stupid" emphasized the concept of simplicity referred to projects and designs; but we believe the concept may very well find application in other fields, inter alia that of surgery.

Acute type A aortic dissection is a dramatic and lethal disease which, when untreated, is associated with a high peri- and intraoperative mortality and morbidity in the setting of emergent surgery.

The primary role of surgery is to prevent the aortic rupture, pericardial tamponade and end-organ malperfusion by resecting the proximal intimal tear restoring the blood flow in the true lumen.

In view of this assessment, the optimal surgical strategy regarding the proximal ascending aorta remains debated.

Many surgeons recommend an extensive replacement of the aortic root (modified Bentall operation) to reduce and/or eliminate the risk of future root dilatation and/or redissection. This option, of course, adds technical complexity and is associated with an increased short-term risk.

On the other hand, a more conservative root repair, such as supracoronary ascending aorta replacement, while representing the easier surgical option with potential less postoperative complications and mortality, leaves the native root with the risk of dilatation and possible future redissection.

Against this backdrop, which is the right surgical operation to opt for in case of acute type A aortic dissection? International literature shows that patients surviving an acute type A aortic dissection have a limited survival in the long-term, that the durability of conservative aortic valveroot replacement-surgery in acute aortic dissection is very often sub-optimal, and that patients which undergo more extensive root replacement operations are not associated with increased hospital mortality (4-6). Moreover, some Authors have shown that conservative and aggressive root management in acute type A aortic dissection reveals similar results both in the early and late mortality, that complete root replacement does not worsen the short-term outcomes, and that both conservative root- and root replacementsurgery can be performed with an acceptable postoperative outcome and late survival (7-9).

Considering the above, under a practical point of view, what would be advisable or ideally right to do in case of acute type A aortic dissection? With the ambitious purpose of offering an answer to these questions, Chiu et al. have performed a retrospective review of the acute type A aortic 
dissection at Stanford Hospital in a period ranging from 2005 to 2015 in which they identified 293 patients without connective tissue disorders who underwent limited root repair (such as aortic valve resuspension, use of biological glue in the dissected root-wall, aortic root remodeling, sinotubular junction reconstruction) or complete replacement of the aortic root with composite valve graft or with valve-sparing aortic root replacement (10).

The results of the study have highlighted that there were no differences both in terms of perioperative mortality as well as in terms of mid-term survival between the two different surgical approaches.

Moreover, they showed that limited root repair was associated with increased risk of late reoperation such as, conversely, the complete root replacement decreases the rate of reoperation (10).

This conclusion basically proceeds from what the data reported in the literature already states: a more extensive root replacement seems to be protective against future proximal aortic reintervention decreasing the rate of late reoperation.

The "take-home" message of the study absolutely agrees with us, especially taking into account the results reported in the literature relating to the two different surgical approaches in acute type A aortic dissection: root replacement and limited root repair. Basically, complicating an intervention which is "per se" already quite complex, does not necessarily grant better results than other less radical surgical options.

The type of approach to be used in emergencies such as a type A dissection depend a lot on the experience of the surgical team, even if, regardless of the surgical technique used and of the radicality of surgery itself, the primary aim of this type of surgery must always be kept in mind: save the patient's life immediately. Regardless of the type of surgical technique used.

Our personal opinion on the strategy to be adopted in this setting depends from the patient in question, because each patient is different. In case of an initial calcified aortic valve, root dilatation or proximal location of the intimal tear, a modified Bentall procedure is justified. In other cases, a supracoronary ascending aorta replacement or a limited root repair can be considered a good option and a good compromise to give the patient a few years of freedom from anticoagulation therapy and prosthesis potential complications.

We must also consider that oftentimes, dissections are operated at night by on-call surgeons with little experience and in these cases and when the general conditions allow it, it would be preferable to perform a supracoronary ascending aorta replacement rather than a valve sparingroot replacement or a modified Bentall operation.

Hence, in light of all of the above considerations, could it be deemed reasonable to apply the "KISS principle" in the planning of the surgical strategy? Our answer is: yes! It must indeed be applied.

Surgical experience plays a very important role in the choice of the correct surgical strategy and technique in the emergent setting of acute type A aortic dissection.

In fact, what history teaches us as to the notion of the "KISS principle" and similar minimalist concepts like Occam's razor which suggests to "eliminate with knife cuts", i.e., the problem-solving principle that the simplest solution tends to be the right one, or Leonardo da Vinci's "Simplicity is the last sophistication", or Mies Van Der Rohe's "Less is more", Bjarne Stroustrup's “Make Simple Tasks Simple!”, or Antoine de Saint Exupéry's known "Perfection is achieved not when there is nothing more to add, but when there is nothing left to take away" ... this should be the path to follow when approaching the surgery of acute dissection type A, always bearing in mind that the most important thing is to save the patient's life.

Therefore, it is the experience of each individual surgeon, along with common sense, which should guide the choice to the type of strategy to be used in situations like these.

To this end, surgeons with limited experience should not feel obliged to perform more radical and complex interventions if they are able to achieve the primary objective with less complex surgery: to save the patient's life.

\section{Acknowledgements}

None.

\section{Footnote}

Conflicts of Interest: The authors have no conflicts of interest to declare.

\section{References}

1. Dalzell T. The Routledge Dictionary of Modern American Slang and Unconventional English. Routledge, London, 2009;595.

2. Partridge E, Dalzell T, Victor T. The Concise New Partridge Dictionary of Slang. Psychology Press, 2007;384. 
3. Rich BR. Clarence Leonard (Kelly). Johnson 1910-1990: A Biographical Memoir. 1995, National Academies Press, Washington, DC, 1995;13.

4. Di Eusanio M, Trimarchi S, Peterson MD, et al. Root replacement surgery versus more conservative management during type A acute aortic dissection repair. Ann Thorac Surg 2014;98:2078-84.

5. Tanaka H, Ikeno Y, Abe N, et al. Outcomes of valvesparing root replacement in acute type A aortic dissection. Eur J Cardiothorac Surg 2018;53:1021-6.

6. Saczkowski R, Malas T, Mesana T, et al. Aortic valve preservation and repair in acute Type A aortic dissection. Eur J Cardiothorac Surg 2014;45:e220-6.

7. Peterss S, Dumfarth J, Rizzo JA, et al. Sparing the aortic

Cite this article as: Di Marco L, Leone A, Murana G, Pacini D. The application of the "KISS principle" for the treatment of type A acute aortic dissection: is this always right? J Thorac Dis 2018;10(Suppl 33):S3884-S3886. doi: 10.21037/jtd.2018.08.132 root in acute aortic dissection type A: risk reduction and restored integrity of the untouched root. Eur J Cardiothorac Surg 2016;50:232-9.

8. Hysi I, Juthier F, Fabre O, et al. Aortic root surgery improves long-term survival after acute type A aortic dissection. Int J Cardiol 2015;184:285-90.

9. Castrovinci S, Pacini D, Di Marco L, et al. Surgical management of aortic root in type $\mathrm{A}$ acute aortic dissection: a propensity-score analysis. Eur J Cardiothorac Surg 2016;50:223-9.

10. Chiu P, Trojan J, Tsou S, et al. Limited root repair in acute type A aortic dissection is safe but results in increased risk of reoperation. J Thorac Cardiovasc Surg 2018;155:1-7.e1. 